\title{
Entire Function Sharing a Small Function with Its Derivatives
}

\author{
Huiliang Zhang, Zongsheng Gao, Yueyang Zhang
}

School of Mathematics and Systems Science, Beihang University, Beijing, China

huilianghang009@163.com

Keywords: Entire functions, Wiman-Valiron theory, Derivatives

\begin{abstract}
In this paper, we mainly apply the Wiman-Valiron theory to prove a theorem on growth of a linear differential equation. From this we obtain a uniqueness theorem concerning that a non-constant entire function and its derivative sharing a small entire function.
\end{abstract}

\section{Introduction}

In this paper, a meromorphic function $f$ always means meromorphic in the whole complex plane. We assume that the reader is familiar with the fundamental results and the standard notions of Nevanlinna's value distribution theory of meromorphic functions (see, e.g., [1], [2]), such as $T(r, f), m(r, f)$. In the whole paper, the notation $S(r, f)$ is defined to be any quantity that satisfies $S(r, f)=o(T(r, f))$ as $r \rightarrow \infty$ outside of a possible exceptional set of finite logarithmic measure. A meromorphic function $a(z)$ is called a small function with respect to $f(z)$ provided that $T(r, a(z))=S(r, f)$.

Let $f$ and $g$ be two non-constant meromorphic functions. We say $\mathrm{f}$ and $\mathrm{g}$ share some finite value a IM (ignoring multiplicities) provided that $f-a$ and $g-a$ have the same zeros.

If $f-a$ and $g-a$ have the same zeros with the same multiplicities, we say that $f$ and g share the value a CM (counting multiplexes). The subject on sharing values between a meromorphic function and its derivative was first studied by Rubel and Yang [5]. They proved that $f=f^{\prime}$ if $f$ and $f^{\prime}$ share two distinct finite constants CM. Mue and Steinmetz[3] pointed out that the same conclusion holds if the two CM shared values are replaced by two IM shared values. For one CM shared value, Brück [9] raised the following famous conjecture which has been well studied.

Conjecture A. (see [9]) Let $\mathrm{f}$ be a non-constant entire function such that $\delta_{2}(f)<1$, and $\delta_{2}(f) \notin \mathrm{N}$. If $f$ and $f^{\prime}$ share a finite value a CM, then $\frac{f^{\prime}-a}{f-a}=c$, where c is a nonzero constant and $\sigma_{2}(f)$ denote the hyper-order of $f$ which is defined by

$$
\sigma_{2}(f)=\limsup _{r \rightarrow \infty} \frac{\log \log T(r, f)}{\log r}=\limsup _{r \rightarrow \infty} \frac{\log \log \log M(r, f)}{\log r}
$$

The case a $=0$ and that $N\left(r, \frac{1}{f}\right)=S(r, f)$ has been proved by Brück [9], while the case that $f$ is of finite order has been proved by Gundersen and Yang [4]. A natural question is that what can be said if a non-constant entire function $\mathrm{f}$ and one of its derivatives $f^{(n)}(n \geq 1)$ share a small entire function of $\mathrm{f}$ ? We first recall the following two theorems related to this question.

Theorem B. (see [7]) Let $f$ be an entire function of finite order and $a$ be an entire function of order less than the order of $\mathrm{f}$. If $f$ and $f^{\prime}$ share a CM, then $f^{\prime}-a=c(f-a)$ for some nonzero constant c. 
Theorem C. (see [6]) If $\mathrm{f}$ is a non-constant solution of the differential equation $f^{(n)}-a_{1}=e^{Q}\left(f-a_{2}\right)$ where $n(\geq 1)$ is a positive integer, $a_{1}$ and $a_{2}$ are two entire functions such that $\sigma\left(a_{j}\right)<1(j=1,2)$, and $Q$ is a polynomial with degree $\operatorname{deg}\{Q\}$, then $\sigma_{2}(f)=q$.

For dealing with the above mentioned question, we prove the following theorem 1.1, which improves the results of Theorem B and Theorem C.

Theorem 1.1 if $\mathrm{f}$ is a non-constant solution of the differential equation

$f^{(n)}-a_{1}=e^{Q}\left(f-a_{2}\right)(1.2)$, where $n(\geq 1)$ is a positive integer, $a_{1}$ and $a_{2}$ are two entire functions such that $\sigma\left(a_{j}\right)<\sigma(f)(j=1,2)$, and $\mathrm{Q}$ is a polynomial with degree $\operatorname{deg}\{Q\}$, then $\sigma_{2}(f)=q$.

From Theorem 1.1, we immediately obtain the following Corollary 1.1.

Corollary 1.1. Let $\mathrm{f}$ be an entire function of finite order and a be an entire function of order less than the order of $\mathrm{f}$, and $\mathrm{n}$ be a positive integer. If $f$ and $f^{(n)}$ share a CM, then $f^{(n)}-a=c(f-a)$ for some nonzero constant c.

\section{Lemmas for the Proofs}

Let $f(z)=\sum_{n=0}^{\infty} a_{n} z^{n}$ be an entire function. Next we define by $u(r, f)=\max \left\{\left|a_{n}\right| r^{n}: n=0,1, \ldots\right\}$ the maximum term of $F(z)$, and define by $v(r, f)=$ $\max \left\{\mathrm{m}: \quad v(r, f)=\left|a_{m} r^{m}\right|\right\}$ the central index of $\mathrm{F}(\mathrm{z})$ (see [2]).

Lemma 2.1. (see [2]) Let $\mathrm{F}$ be an entire function of order $\sigma(\mathrm{F})=\sigma$, and let $v(r, F)$ be the central index of F. Then $\limsup _{r \rightarrow \infty} \frac{\log v(r, f)}{\log r}=\sigma$

The following Lemma 2.2 is well known as the Wiman-Varilon theory and is a useful device when considering the value distribution of entire functions of complex differential equations.

Lemma 2.2. (see [2]) Let $F$ be a transcendental entire function, and let $0<\delta<\frac{1}{4}$ and z be such that $|z|=r$ and that

$$
|\mathrm{F}(\mathrm{z})|>\mathrm{M}(\mathrm{r}, \mathrm{F}) \mathrm{v}(\mathrm{r}, \mathrm{F})^{-\frac{1}{4}+\delta}
$$

holds. Then there exists a set $E \subset R_{+}$of finite logarithmic measure such that

$$
F^{(n)}(z)=\left(\frac{v(r, F)}{z}\right)^{n}(1+o(1)) F(z)
$$

holds for all $\mathrm{m} \geq 0$ and all $\mathrm{r} \notin \mathrm{E}$

Lemma 2.3. Let $F$ be a transcendental entire function satisfying $\sigma(F)=\sigma<\infty$, and let $0<\delta<$ $1 / 4$ and $\mathrm{z}$ be such that $|\mathrm{z}|=\mathrm{r}$ such that (2.1) holds. Then for any given $\varepsilon>0$, there exists a set $E \subset R_{+}$of finite logarithmic measure such that

$$
|z|^{n(1-\sigma-\varepsilon)} \leq\left|\frac{F(z)}{F^{n}(z)}\right| \leq(1+o(1))|z|^{n}
$$

holds for all $\mathrm{n} \geq 0$ and all $\mathrm{r} \notin \mathrm{E}$ as $|\mathrm{z}|=\mathrm{r} \rightarrow \infty$.Moreover, there exists an infinite sequence $Z_{m}=r_{m} e^{\theta_{m}}, \theta_{m} \in[0,2 \pi)$ satisfying $\lim _{m \rightarrow \infty} \theta_{m}=\theta_{0}$ and some constant $R=R(\varepsilon)>0$ and $\eta(\varepsilon)>0$ such that $\left|F^{n}(z)\right| \geq \frac{e^{r_{m}{ }^{\sigma-\varepsilon}}}{4 r_{m}{ }^{n}}$ holds for all $z$ satisfying $|z|=r_{m} \geq R$ and $\arg z \in\left[\theta_{0}-\eta, \theta_{0}+\eta\right]$ 
Proof Let $v(r, F)$ be the central index of $\mathrm{F}$, and let $0<\delta<\frac{1}{4}$ and $z$ be such that $|z|=r$ and that (2.1) holds. Then by Lemma 2.2, we know that there exists a set $\mathrm{E} \subset R_{+}$of finite logarithmic measure such that (2.2) holds for all holds for all $n \geq 0$ and all $r \notin E$. Since $\frac{1}{1+o(1)}$ is also of type1+o(1), we obtain from (2.2) that $\frac{F(z)}{F^{n}(z)}=\left(\frac{Z}{v(r, F)}\right)^{n}(1+o(1))$.

From Lemma 2.1, we know that $\lim _{r \rightarrow \infty} \sup \frac{\log v(r, F)}{\log r}=\sigma$, which yields that $v(r, F) \leq r^{\sigma+\varepsilon}$ for any given $\varepsilon>0$ as $r \rightarrow \infty$. Hence $\frac{r}{v(r, F)} \geq r^{1-\sigma-\varepsilon}$ as $r \rightarrow \infty$. Noting that $v(r, F) \rightarrow \infty$ as $r \rightarrow \infty$, we easily obtain that

$(1+o(1))|z|^{n(1-\sigma-\varepsilon)} \leq\left|\frac{F(z)}{F^{n}(z)}\right| \leq(1+o(1))|z|^{n}$ as $r \rightarrow \infty$, which is (2.3). Since $0<\sigma<\frac{1}{4}$ and $V(r, F) \rightarrow \infty$ as $r \rightarrow \infty$, we have $v(r, F)^{-\frac{1}{4}+\delta} \rightarrow 0$ as $r \rightarrow \infty$. This gives that there exists some $R_{0}>0$ such that $v(r, F)^{-\frac{1}{4}+\delta}<\frac{1}{2}$ where $r>R_{0}$. For the given $\varepsilon>0$, let $z_{m}=r_{m} e^{i \theta_{m}}, \theta_{m} \in[0,2 \pi)$ be such that $\left|f\left(z_{m}\right)\right|=M\left(r_{m}, F\right) \geq e^{r_{m} \sigma-\varepsilon}$ on $\left|z_{m}\right|=r_{m}$. Obviously, there is a subset $\left\{\theta_{m_{k}}\right\}$ of $\left\{\theta_{m}\right\}$ satisfying $\lim _{m_{k} \rightarrow \infty} \theta_{m_{k}}=\theta_{0} \in[0,2 \pi)$. Without loss of generality, we suppose that $\lim _{m \rightarrow \infty} \theta_{m}=\theta_{0}$. Then there exists some $R=R(\varepsilon)>R_{0}$ and some $\eta=\eta(\varepsilon)>0 \quad$ independent of $r$ such that $|F(z)|>\frac{1}{2} M\left(r_{m}, F\right) \geq \frac{1}{2} e^{r_{m}{ }^{\sigma-\varepsilon}}$ on $|z|=r_{m}$ and $\arg z \in\left[\theta_{0}-\eta, \theta_{0}+\eta\right]$ for all $r>R$. From (2.3), we immediately get $\left|F^{(n)}(z)\right| \geq \frac{|F(z)|}{(1+o(1))|z|^{n}}$, which yields that $\left|F^{n}(z)\right| \geq \frac{e^{r_{m}{ }^{\sigma-\varepsilon}}}{4 r_{m}{ }^{n}}$ on $|z|=r_{m}$ and $\arg z \in\left[\theta_{0}-\eta, \theta_{0}+\eta\right]$ for all $|z|=r_{m}>R$.

Lemma 2.4. (see [8]) Let $B(z)=b_{n} z^{n}+b_{n-1} z^{n-1}+\ldots+b_{0}$ be a polynomial, where $n$ is a positive integer and $n$ is a positive integer and $b_{n}=\alpha_{n} e^{i \theta_{n}}, \theta_{n} \in[0,2 \pi)$, For any given $0<\varepsilon<\frac{\pi}{4 n}$, we introduce $2 \mathrm{n} \quad$ closed angles $S_{j}: \frac{-\theta_{n}}{n}+(2 j-1) \frac{\pi}{2 n}+\varepsilon<\theta<\frac{-\theta_{n}}{n}+(2 j+1) \frac{\pi}{2 n}-\varepsilon(j=0,1, \ldots, 2 n-1)$.Then there exists a positive number $R=R(\varepsilon)$ such that for $|z|=r<R, \operatorname{Re}\{Q(z)\}>-\alpha_{n}(1-\varepsilon) \sin (n \varepsilon) r^{n}$. If $z \in S_{j}$, where $j$ is even; while $\operatorname{Re}\{Q(z)\}<-\alpha_{n}(1-\varepsilon) \sin (n \varepsilon) r^{n}$ If $z \in S_{j}$, where $j$ is odd.

Lemma 2.5. (see [10]) Let $\mathrm{F}$ be an entire function of infinite order, with the hyper-order $\sigma_{2}(F)=\sigma_{2}$, and let $v(r, F)$ and let $v(r, F)$ be the central index of $\mathrm{F}$. Then $\limsup _{r \rightarrow \infty} \frac{\log \log v(r, F)}{\log r}=\sigma_{2}$

\section{Proof of Theorem 1.1}

Set $F=f-a_{2}$, then $\mathrm{F}$ is an entire function and $f^{(n)}=F^{(n)}+a_{2}^{(n)}$, substituting $f=F+a_{2}$ and $f^{(n)}=F^{(n)}+a_{2}^{(n)}$ into (1.2), we get 


$$
F^{(n)}=e^{Q(z)} F+a_{1}-a_{2}^{(n)}
$$

Denote $b=a_{1}-a^{(n)}$, then we have

$$
F^{(n)}=e^{Q(z)} F+b
$$

Obviously, b is a finite order entire function and $\sigma(b) \leq \max \left\{\sigma\left(a_{1}, a_{2}\right)\right\}<\sigma(f)=\sigma(F)$. It is well known that all solutions of (3.2) are entire functions. We then prove the following cases:

Case 1: $\mathrm{Q}$ is a constant, then $e^{Q}=c \neq 0$. Thus we can write (3.2) as

$$
F^{(n)}=c F+b
$$

We claim that $\mathrm{F}$ is an entire function of finite order. Otherwise, we suppose that $\sigma(F)=\infty$.Let $0<\delta<\frac{1}{4}$ and $\mathrm{z}$ be such that $|z|=r$ and that (2.1) holds. Then by Lemma 2.2 there exists a set $E \subset R_{+}$of finite logarithmic measure such that (2.2) holds for all $\mathrm{n} \geq 0$ and all $r \notin E$. Hence we have

$$
\left(\frac{v(r, F)}{Z}\right)^{m}(1+o(1))=c+\frac{b}{F}
$$

Note that $\mathrm{F}$ is an entire function of infinite order. For any given constant $\mathrm{N}>0$, let $z_{m}=r_{m} e^{i \theta_{m}}, \theta_{m} \in[0,2 \pi)$ be such that $\left|F\left(z_{m}\right)\right|=M\left(r_{m}, F\right)$ on $\left|z_{m}\right|=r_{m}$

By Lemma 2.5, we know that there is an infinite sequence $\left\{r_{m}{ }^{\prime}\right\}$ such that

$$
\lim _{r_{m} \rightarrow \infty} \frac{\log \log v\left(r_{m}, F\right)}{\log r_{m}^{\prime}}=\sigma_{2}(F) \text {. }
$$

Set the logarithmic measure of $\mathrm{E}, \operatorname{ImE}=\delta<\infty$, then there is a point $r_{m} \in\left[r_{m}^{\prime},(\delta+1) r_{m}{ }_{m}\right) / E$, since

$$
\frac{\log \log v\left(r_{m}, F\right)}{\log r_{m}} \geq \frac{\log \log v\left(r_{m}^{\prime}, F\right)}{\log (\delta+1) r_{m}^{\prime}}=\frac{\log \log v\left(r_{m}^{\prime}, F\right)}{\log r_{m}^{\prime}\left[1+\frac{\log (\delta+1)}{\log r_{m}^{\prime}}\right]},
$$

We have $\lim _{r_{m} \rightarrow \infty} \frac{\log \log v\left(r_{m}, F\right)}{\log r_{m}}=\sigma_{2}(F)$.

Obviously, we have $\lim _{r_{m} \rightarrow \infty} \frac{\log v\left(r_{m}, F\right)}{\log r_{m}}=\infty$

From (3.5), we obtain that $r_{m}^{N} \leq v\left(r_{m}, F\right) \leq e^{r^{\sigma_{2}+\varepsilon}{ }_{m}}$ for any $\varepsilon>0$ and any big real constant $N>0$ as $r_{m} \rightarrow \infty$.For convenience, we denote all such points by $\left\{z_{m}\right\}$. Since $\sigma(F)=\infty$, then there exists a subset $\left\{z_{j}\right\}$ of $\left\{z_{m}\right\}$ satisfying $M\left(r_{j}, F\right) \geq e^{r^{\sigma_{0+1}{ }_{j}}}$ as $r_{j} \rightarrow \infty$. Denote $\sigma(b)=\sigma_{0}$, then for the given $\varepsilon>0$, we have $\left|b\left(z_{j}\right)\right| \leq e^{r^{\sigma_{0+\varepsilon}}{ }_{j}}$ as $r_{j} \rightarrow \infty$. Let $N>\max \left\{1, \sigma_{0}+\varepsilon\right\}$. We see that the module of the right hand side of (3.4) tends to $|\mathrm{c}|$ as $r_{j} \rightarrow \infty$ while the module of the left hand side of (3.4) tends to $|c|$ as $r_{j} \rightarrow \infty$, which is impossible. Thus we obtain that F must be of finite order, and therefore $\sigma_{2}(f)=\sigma_{2}(F)=\operatorname{deg}\{Q\}=0$.

Case 2: $Q(z)$ is a non-constant polynomial with $\operatorname{deg}\{Q(z)\}=q \geq 1$. We first proving that $\mathrm{F}$ is of infinite order. Otherwise, since $Q(z)$ is a non-constant polynomial with $\operatorname{deg}\{Q(z)\}=q \geq 1$, we 
may suppose that $\mathrm{F}$ is an entire function of finite order satisfying $1 \leq \sigma(F)=\sigma<\infty$, we rewrite (3.2) as the following form

$$
e^{Q(z)} \frac{F}{F^{(n)}}=1-\frac{b(z)}{F^{(n)}}
$$

Let $0<\delta<\frac{1}{4}$ and $\mathrm{z}$ be such that $|z|=r$ and that (2.1) holds, then by Lemma2.3, there exists a set $E \subset R_{+}$of finite logarithmic measure such that

$$
(1+0(1))|z|^{n(1-\sigma-\varepsilon)} \leq\left|\frac{F(z)}{F^{n}(z)}\right| \leq(1+o(1))|z|^{n}
$$

For all $r \notin E$ as $r \rightarrow \infty$, Moreover, for any given $\varepsilon>0$, there exists an infinite sequence $\quad z_{m}=r_{m} e^{\theta_{m}}, \theta_{m} \in[0,2 \pi) \quad$ satisfying $\quad \lim _{m \rightarrow \infty} \theta_{m}=\theta_{0} \in[0,2 \pi) \quad$ and some constants $R_{0}=R(\varepsilon)>0$ and $\eta=\eta(\varepsilon)>0$ such that $\left|F^{(n)}(\mathrm{z})\right| \geq \frac{e^{r_{m}{ }^{\sigma-\varepsilon}}}{r_{m}{ }^{n}}$ holds for all $\mathrm{z}$ such that $|z|=r_{m} \geq R_{0}$ and $\arg z \in\left[\theta_{0}-\eta, \theta_{0}+\eta\right]$. Note that $|b(z)| \leq e^{r^{\sigma_{0}+\varepsilon}{ }_{m}}$ as $r_{m} \rightarrow \infty$. Hence we have $\left|\frac{b(z)}{F^{(n)}(z)}\right| \leq \frac{r_{m}^{n} e^{r_{m}^{\sigma_{0+\varepsilon}}}}{e^{r_{m}^{\sigma_{0}-\varepsilon}}}$ Let $0<\varepsilon<\frac{\sigma-\sigma_{0}}{2}$, then from the above equation we obtain that the module of right hand side of equation (3.6) tends to 1 as $r \rightarrow \infty$. On the other hand, denote $Q(z)=\alpha_{q} z^{q}+\ldots$ and $\alpha_{q}=\left|\alpha_{q}\right| e^{i \theta_{q}}$, then from Lemma 2.4, we know that for the given $\varepsilon$ satisfying $0<\varepsilon$ $<\min \left\{\frac{\pi}{4 q}, 2 \eta, \frac{\sigma-\sigma_{0}}{2}\right\} \quad$, if $\quad$ we introduce $2 q$ closed angles $S_{t}: \frac{-\theta_{q}}{q}+(2 t-1) \frac{\pi}{2 q}+\varepsilon<\theta<\frac{-\theta_{q}}{q}+(2 t+1) \frac{\pi}{2 q}-\varepsilon(t=0,1, \ldots, 2 n-1)$,

Then there exists a positive number $R_{1}=R(\varepsilon)$ such that for $|z|=r>R_{1}$,

$\operatorname{Re}\{Q(z)\}>\left|\alpha_{q}\right|(1-\varepsilon) \sin (q \varepsilon) r^{q}$

If $z \in S_{t}$, where $t$ is even; while

$$
\operatorname{Re}\{Q(z)\}<-\left|\alpha_{q}\right|(1-\varepsilon) \sin (q \varepsilon) r^{q}
$$

If $z \in S_{t} \quad$ where $\mathrm{t}$ is odd. Thus for all $|z|=r_{m} \notin E$, when $|z|=r_{m}$ satisfying $r_{m}>\max \left\{R_{0}, R_{1}\right\}$ and $\arg z \in\left[\theta_{0}-\eta, \theta_{0}+\eta\right]$, if $z \in S_{t}$, where $\mathrm{t}$ is even, from (3.7) and (3.8) we have $\left|e^{Q(z)} \frac{F}{F^{(n)}}\right| \geq e^{\left|\alpha_{q}\right| \sin (q \varepsilon) r_{m}{ }^{q}}(1+o(1))|z|^{n(1-\sigma-\varepsilon)}$, a contradiction to the right hand side of (3.6) when we let $r_{m} \rightarrow \infty$, if $z \in S_{t}$, where $t$ is odd and $\arg z \in\left[\theta_{0}-\eta, \theta_{0}+\eta\right]$, from (3.7) and (3.9) we have $\left|e^{Q(z)} \frac{F}{F^{(n)}}\right| \leq e^{-\left|\alpha_{q}\right| \sin (q \varepsilon) r_{m}{ }^{q}}(1+o(1))|z|^{n}$, a contradiction to the right hand side of (3.6) when we let $r_{m} \rightarrow \infty$. Hence $F(z)$ must be of infinite order. Now we prove that $\sigma_{2}(F)=\sigma_{2}=\operatorname{deg}\{Q\}=q$, let $0<\delta<\frac{1}{4}$ and $z$ be such that (2.1) holds on $|z|=r$. Then there exists a set $E \subset R_{+}$of finite logarithmic measure such that (2.2) holds for all $n \geq 0$ and all $r \notin E$. Note that $F$ is an entire function of infinite order. For any constant $M>0$, let $z_{m}=r_{m} e^{i \theta_{m}}, \theta_{m} \in[0,2 \pi)$ be such that $\left|f\left(z_{m}\right)\right|=M\left(r_{m}, F\right) \geq e^{r_{m}{ }^{M}}$ on $\left|z_{m}\right|=r_{m}$. From the above reasoning, we may suppose 
that $\lim _{r_{m} \rightarrow \infty} \theta_{m}=\theta_{0} \in[0,2 \pi)$ and that the infinite sequence $\left\{r_{m}\right\}$ satisfies (3.5), then for any $\varepsilon>0$, we have

$r_{m}{ }^{M} \leq v\left(r_{m}, F\right) \leq e^{r_{m} \sigma_{2}+\varepsilon}$ as $m \rightarrow \infty$, By Lemma 2.2 and (3.2) we have

$$
\left(\frac{v(r, F)}{z}\right)^{n}=e^{Q(z)}+\frac{b(z)}{F}
$$

Since $|b| \leq e^{r^{\sigma_{0}+\varepsilon}}$ as $r \rightarrow \infty$, let $M>\sigma_{0}+\varepsilon$, then we obtain from (3.10) that

$$
\left|\frac{v\left(r_{m}, F\right)}{z_{m}}\right|^{n} \leq 2\left(\left|e^{Q\left(z_{m}\right)}\right|+1\right) \leq 2 e^{r_{m}{ }^{q+\varepsilon}}
$$

Since $\varepsilon$ is arbitrary, then from (3.11) and Lemma 2.5 we obtain that $\sigma_{2}<q$. Now we suppose that $\sigma_{2}<q$. Note that there exists some $R_{2}>0$ such that $v(r, F)^{-\frac{1}{4}+\delta}<\frac{1}{2}$ when $r>R_{2}$, then for the infinite sequence $z_{m}=r_{m} e^{i \theta_{m}}$ satisfying

$\lim _{r_{m} \rightarrow \infty} \theta_{m}=\theta_{0} \in[0,2 \pi)$, there exists some $R_{3}>R_{2}$ and some $\eta>0$ independent of $r$ such that $|f(z)|>\frac{1}{2} M\left(r_{m}, f\right) \geq \frac{e^{r_{m}{ }^{M}}}{2}$ on $|z|=r_{m} \quad$ and $\quad \arg z \in\left[\theta_{0}-\eta, \theta_{0}+\eta\right] \quad$ for $\quad$ all $\quad r_{m}>R_{3}$. Then $\left|\frac{b(z)}{F(z)}\right| \rightarrow 0$ as $r \rightarrow \infty$ in (3.10). Then from (3.10), we can get that

$$
r_{m}^{n(M-1)} \leq\left|e^{Q(z)}\right| \leq \frac{e^{n r_{m}{ }^{\sigma_{2}+\varepsilon}}}{r_{m}{ }^{n}}+o(1)
$$

Let $M>1$ and select $\varepsilon>0$ satisfying $0<\varepsilon<\left\{\frac{q-\sigma_{2}}{2}, 2 \eta, \frac{\pi}{4 q}\right\}$, we see that the above equation contradicts with (3.8) and (3.9) when $|z|=r_{m}$ is large enough. This leads to that $\sigma_{2}=q$.We complete the proof.

\section{References}

[1] C.C. Yang, H.X. Yi, Uniqueness Theory of Meromorphic Function, Dordrecht: Kluwer, 2003.

[2] I. Laine, Nevanlinna Theory and Complex Differential Equations, Berlin de Gruyter,1993.

[3] E. Mues und N. Steinmetz, Meromorphe Funktionen, die mit ihrer Ableitung Werteteilen, Manuscripta Math. 29 (1979), 195-206.

[4] G. G. Gundersen, L. Z. Yang, Entire functions that share one value with one or two of their derivatives, J. Math. Anal. Appl. 223 (1998), 88-95.

[5] L. A. Rubel and C. C. Yang, Values shared by an entire function and its derivative, Lecture Notes in Math. English Series 599 (1977), 101-103.

[6] J. Wang and X. M Li, The Uniqueness of an entire function sharing a small entire function with its derivative, J. Math. Anal. Appl. 354 (2009), 478-489.

[7] J.M. Chang and Y.Z.Zhu, Entire functions that share a small function with their derivatives, J. Math. Anal. Appl. 351 (2009), 491-496.

[8] Markushevich A. I., Theory of Functions of a Complex variable, Prentice-Hall, New Jersey, 1995. 
[9] R. Bruck, On entire functions which share one value CM with their first derivative, Results Math. 30 (1996), 21-24.

[10] Z.X. Chen, on the hyper-order of solutions of some second order linear differential equations, Acta Math Sinica, English Series 18(1) (2002), 79-88. 